\title{
Left ventricular systolic function assessment in patients with dilated heart failure using cardiovascular magnetic resonance
}

\author{
Eddie Y.K. $\mathrm{Ng}^{1}$ \& Wan Kee $\mathrm{Ng}^{2}$ \\ ${ }^{1}$ School of Mechanical and Aerospace Engineering, College of Engineering, Nanyang Technological University, 50 Nanyang Avenue, Singapore 639798. ${ }^{2}$ The \\ Australian School of Advanced Medicine, Macquarie University, Sydney, NSW 2109, Australia. Correspondence should be addressed to Ng* E.Y.K. \\ (mykng@ntu.edu.sg).
}

Received June 6, 2008; revised October 22, 2008; accepted October 22, 2008

\begin{abstract}
Cardiovascular magnetic resonance (CMR) has become a reference standard for the measurement of cardiac volumes, function, and mass. This study aims to reconstruct three dimensional modeling of the left ventricle (LV) in patients with heart failure (HF) using CMRtools and thereby derive the LV functional indices. CMR images were acquired in 41 subjects ( 6 females) with heart failure (HF) and 12 normal controls (4 females). Five comparisons were made (i) normal and dilated heart failure subjects, (ii) male and female normal heart, (iii) male and female dilated heart, (iv) male normal and dilated heart failure and (v) female normal and dilated heart failure. In HF, a significant higher values of EDV (320 \pm 79 vs. $126 \pm 22 \mathrm{ml}, \mathrm{P}<0.0001)$, ESV (255 \pm 68 vs. $54 \pm 12 \mathrm{ml}, P<0.00001)$ and lower values of EF ( $20 \pm 7$ vs. $58 \pm 5 \%)$ were found compared that of normal control. There were significant difference on LV EDV and ESV between sex in both normal and HF subjects.
\end{abstract}

Keywords: Dilated heart failure, magnetic resonance imaging, left ventricle, systolic function

\section{INTRODUCTION}

The heart is divided into right and left sides by a septum, which is a partition consisting of myocardium covered in endocardium. Each side is further divided by the atrioventricular valve (AV) into upper chamber, the atrium, and lower chamber, the ventricle. The AV valves are formed by double folds of endocardium strengthened by fibrous tissues. The right atrioventricular valve (tricuspid valves) has three flaps or cusps and the left atrioventricular valve (mitral valve) has two.

The heart, with its action as a pump in mechanical analogy, produces of series of events within a period of time known as the cardiac cycle. During each cardiac cycle, the heart contracts and then relaxes creating the systolic and diastolic pressures. Contraction of the heart expels a percentage of oxygenated blood known as the cardiac output, given as product of heart rate and stroke volume. The period of contraction is called systole and that of relaxation, diastole.

The valves between the atria and ventricles open and close passively according to the pressure in the heart chambers. They open when the pressure in the atria is greater than that in the ventricles. During ventricular systole (i.e., contraction), the pressure in the ventricles rises greater than in the atria, thus shuts the valves to prevent backward flow of blood. The valves are prevented from opening upwards into the atria by tendinous cords, which extend from the inferior surface of the cusps to little projections of myocardium covered with endothelium, called the papillary muscles.

The muscle layer of the walls of the atria is very thin compared to the ventricles. This is consistent with the amount of work it does. The atria, assisted by gravity, only propel blood through the AV valves into the ventricles. The ventricles on the other hand, actively pump the blood to the lungs and to the distal parts of the body. In the case of the left ventricle, oxygenated blood from the left atria enters the left AV valve into the left ventricle, and from there the blood is pumped via the aorta, then the peripheral arteries and to different organs. Therefore the muscle layer is thickest in the wall of the left ventricle.

When the pressure developed in the left ventricle by the contraction of myocardium is less than the pressure in the aorta, the ventricle cannot pump out the normal amount of blood resulting in left ventricular failure. This phenomenon can be caused by excessively high systemic (aortic) blood pressure, incompetence of the mitral and/or the aortic valve, aortic valve stenosis and myocardial weakness. Failure of the left ventricle leads to dilatation of the atrium and an increase in pulmonary blood pressure. This is followed by a rise at the blood pressure in the right side of the heart and eventually systemic venous congestion.

1.1. Comparison of Imaging Techniques on Cardiovascular Disease Diagnosis 
Over the past decades, the ability of technology to diagnose heart disease has improved dramatically, largely due to the evolution of new techniques such as electrocardiography, nuclear cardiology, cardiac computed tomography and cardiovascular magnetic resonance [1]. When patients complain of signs and symptoms such as chest pain, shortness of breath, and an abnormal pulse, further diagnosis can then be administered. Further examinations and quantification of the left ventricular (LV) ejection fraction (LVEF), LV end-diastolic volume (LVEDV), and LV end-systolic volume (LVESV). These are important prognostic parameters in patients with chronic coronary artery disease (CAD) and LV dysfunction [2]. Accurate assessment of LVEF and LV volumes in these patients is important, and several imaging techniques are available for this purpose such as the echocardiography, cardiovascular magnetic resonance, Nuclear Cardiology and computed tomography. The advantage and disadvantage of these methods are briefly discussed as follow.

\subsection{Echocardiography Methodology}

Echocardiography is one the noninvasive techniques used in the diagnosis of heart disease. Echocardiograms are obtained by reflecting high frequency sound waves off various structures of the heart, then translating the reflected waves into one- and two-dimensional images. Echocardiography can produce detailed three-dimensional images of the heart's anatomy [7, 10]. These images can used to estimate heart size, functionality, and wall thickness of the muscle. With the combination of Doppler technique, echocardiography can be used to estimate blood flow through the heart chambers and the pressure gradients across valves to determine the degree of narrowing, regulation, or ventricular calcification. When combined with stress test, echocardiography is able to evaluate wall motion of the ventricles and other physical characteristics of the heart under stress.

Echocardiography can also be used to detect tumours or clots within the heart and other congenital abnormalities. It is completely non contact, therefore eliminates any pain or risk during testing. The concern of using this technique is that, echocardiography cannot measure ejection fraction as precise as other imaging techniques especially for patients with broad chests or obese $[8,11]$.

\subsection{Nuclear Cardiology Methodology}

The use of radioactive substances to examine the function of the heart was first introduced as early as 1927 [1]. The analysis was by injecting a small amount of a short-lived radioisotope into the bloodstream and then track its progress and specific uptake in the circulatory system using a radiation-detecting device. During nuclear cardiology procedures, a scintillation gamma camera is used to detect the radiation (gamma rays) emitted by the isotope, the data is collected and processed by computer. Information is then quantified to display as a picture of the heart.

Nuclear cardiology provides accurate measurement of heart function especially the ejection fraction [2, 4, 5]. However, quality of data can be affected for patients with irregular heart rhythm. This technique is not suitable for pregnant women and nursing mother.

\subsection{Cardiac Computed Tomography}

Cardiac Computed Tomography is also known as single photon emission computed tomography (SPECT). SPECT can be used to obtain three-dimensional thallium images of the heart. It is superior in detecting individual lesions in the coronary arteries and to identify the location of damaged and ischemic heart muscle. SPECT has been used to assess the effects of treatment for ischemic heart disease [9]. Although Cardiac Computed tomography is also used to diagnose stroke, its use in cardiovascular disease is mainly confined to diseases of the aorta. The cost of using SPECT is high compared to other standard nuclear imaging techniques.

\subsection{Cardiovascular Magnetic Resonance}

Cardiovascular magnetic resonance (CMR) images are acquired with patient in supine position by using the 1.5-T MRI system with a flexible body array coil for signal reception. Spin-echo scout images are obtained in the coronal and sagittal imaging planes. It enables CMR to provide accurate, reproducible assessment of cardiac function through acquisition of tomography images of high spatial and temporal resolution. CMR is free of ionizing radiation.

LV functional indices will be analysed in this study. They are (i) end-diastolic Volume (EDV), (ii) end-systolic volume (ESV), (iii) ejection fraction (EF) and (iv) stroke volume (SV). They are defined as:

End-diastolic volume (EDV) - EDV is the volume of blood in the ventricle at the end of filling (i.e., end-diastole). An increase in EDV increases the amount of blood ejected from the ventricle during systole (i.e., stroke volume).

End-systolic volume (ESV) - ESV is the volume of blood in the ventricle at the end of the cardiac ejection period and immediately preceding the beginning of ventricular relaxation. Measurement of the adequacy of cardiac emptying relates to systolic function. End-systolic volume will be seen at the end of the $\mathrm{T}$ wave.

Ejection fraction (EF) - EF is the fraction of blood ejected by the ventricle relative to its end-diastolic volume (i.e., $=\mathrm{SV} / \mathrm{EDV} \times 100 \%$ ).

Ejection fraction is commonly measured using echocardiography. This non-invasive technique provides good estimates of EDV, ES,, and SV. Normally, EF is $>60 \%$. EF is often used as a clinical index to evaluate the inotropic status of the heart. A high EF could indicate the presence of certain heart conditions, such as hypertrophy cardiomyopathy [1]. A low EF could be a sign that the heart is weakened [1]. One should be aware that, it is important to note that there are circumstances in which EF can be normal, yet the ventricle is in failure. Oneexample is diastolic dysfunction caused by hypertrophy in 


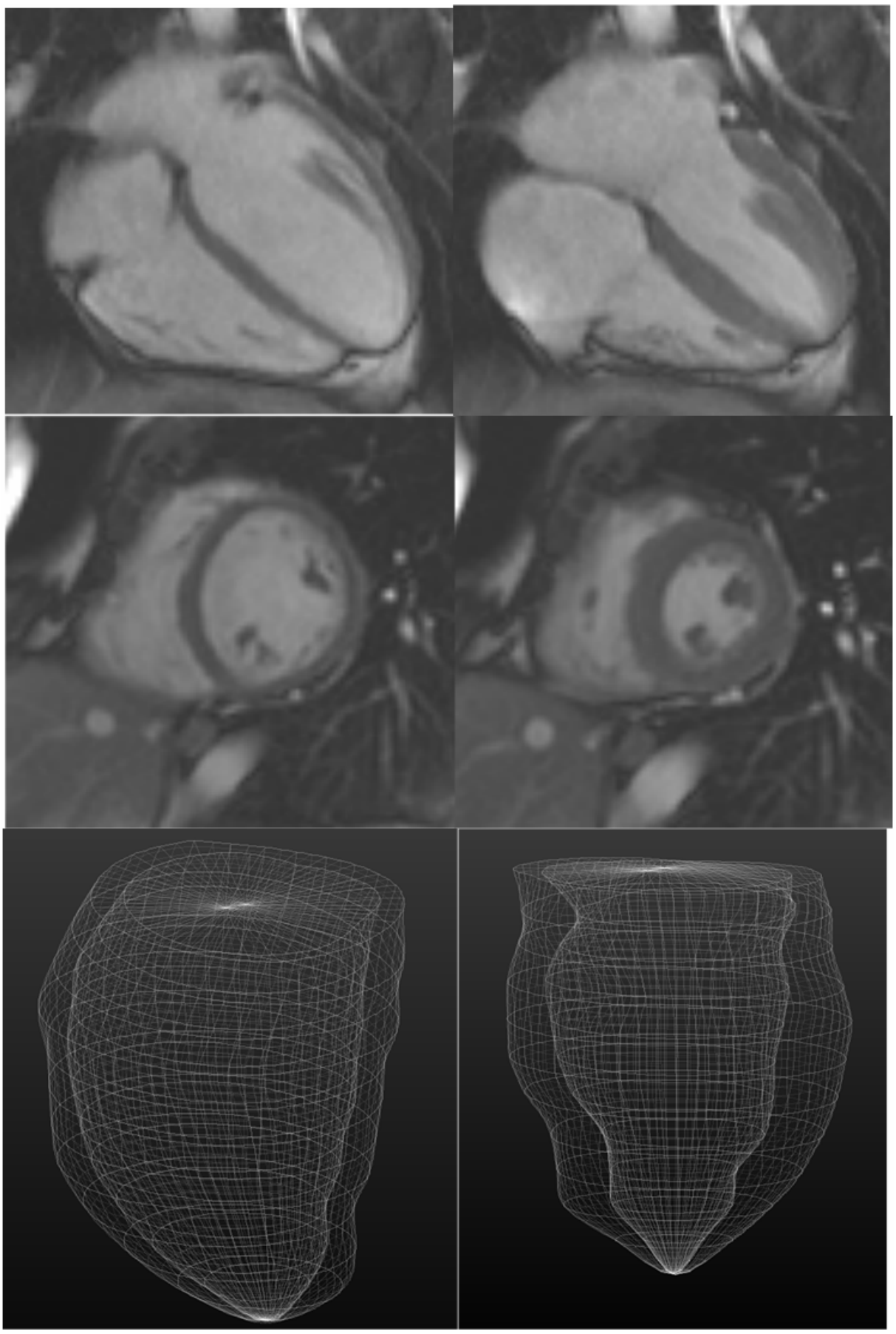

Figure 1. Cine-frame images of a 4-chamber (above panel), a short-axis (middle panel) and 3-dimensional reconstruction (below panel) of left ventricle at end-diastolic (left panel) and end-systolic (right panel) phases. 3-dimensional reconstruction is for calculation of ventricular volumes and function.

Table 1. LV functional Indices between the normal and HF Subjects.

\begin{tabular}{l|llll|llll}
\hline & Normal & & & Heart failure & & \\
\hline & EDV (ml) & ESV (ml) & SV (ml) & EF (\%) & EDV (ml) & ESV (ml) & SV (ml) & EF (\%) \\
Mean \pm SD & $126 \pm \mathbf{2 2}$ & $54 \pm \mathbf{1 3}$ & $73 \pm \mathbf{1 2}$ & $58 \pm \mathbf{5}$ & $320 \pm \mathbf{7 9 *}$ & $255 \pm \mathbf{6 8 *}$ & $65 \pm \mathbf{1 4}$ & $20 \pm 7 *$ \\
Min & 81 & 30 & 51 & 50 & 156 & 101 & 14 & 6 \\
Max & 156 & 78 & 99 & 66 & 489 & 441 & 133 & 38 \\
\hline
\end{tabular}


Table 2. The $P$ values of comparisons.

\begin{tabular}{|l|l|l|l|l|l|}
\hline & & EDV (ml) & ESV (ml) & SV (ml) & EF (\%) \\
\hline \multirow{4}{*}{ P value } & Normal vs. HF & $<0.0001$ & $<0.0001$ & 0.224 & $<0.0001$ \\
\cline { 2 - 6 } & Normal vs. HF in male & $<0.0001$ & $<0.0001$ & 0.062 & $<0.0001$ \\
\cline { 2 - 6 } & Normal vs. HF in female & 0.001 & 0.001 & 0.516 & 0.001 \\
\cline { 2 - 6 } & Male vs. Female in normal & 0.012 & 0.066 & 0.002 & 0.965 \\
\cline { 2 - 6 } & Male vs. Female in HF & 0.004 & 0.010 & 0.224 & 0.635 \\
\hline
\end{tabular}

which filling is impaired because of low ventricular compliance and stroke volume is therefore reduced. In the case of dilated HF, the value of EF be comes very small as SV decreases and EDV increases. In severe HF, EF may be $20 \%$ or less.

Stroke volume (SV) - SV is the amount of blood pumped by the LV in one contraction. Normally, only about two-thirds of the blood in the ventricle is pumped out with each beat. What blood is actually pumped from the left ventricle is the stroke volume and it, together with the heart rate, determines the cardiac output.

\section{METHOD AND DESIGN}

53 subjects were included in the study from two groups: 41 patients with dilated HF and 12 normal volunteers. CMRtools was used to reconstruct the 3-dimensional (3D) model of the LV and to derive LV functional indices (i.e., EDV, ESV, SV and EF). One sample 3D model of the LV was shown in Figure 1. 2-sample t-test was performed to assess any significant differences on LV functional indices between normal and dilated HF subjects. A commercially available statistical software package was used for data analysis (Minitab 14).

\section{RESULTS}

LV Functional Indices for Normal and HF Subjects are summarized in Table 1. EDV and ESV in HF are significantly higher than that of the normal control. LV EF is significant lower that that of the normal control. The detail analysis by using 2-sample t-test between the normal control and dilated HF subjects on EDV, ESV, SV and EV are summarized in Table 2.

In normal subjects, there is statistical significant difference on EDV and SV between male and female, but not for ESV and EF. In HF subjects, there is statistical significant difference on EDV and ESV between male and female. It was seen that in both the normal and dilated HF, male had higher values of EDV. When it comes to ESV, both male and female have the same value in the normal subjects. However, male has higher ESV values in HF subjects. SV, on the other hand, shows reversed trend. Male subjects show higher SV compared to female in the normal subjects, however, there is no significant difference between SV in HF subjects. There is no significant difference between male and female in EF in both the normal and HF subjects.

\section{DISCUSSION}

LV 3-dimensional modeling was reconstructed and the $\mathrm{LV}$ functional indices were derived. It was seen that the mean value of EDV is $126 \mathrm{ml}$ in normal subjects compared to $320 \mathrm{ml}$ in the dilated HF subjects For ESV, the values increased from $54 \mathrm{ml}$ in normal subjects to $255 \mathrm{ml}$ in dilated HF subjects.

Various studies have been done to compare the functional indices between MRI and various other medical imaging available when diagnosing the normal and heart failure [2, 3, 4, 6, 8, 12]. Riemer et al [2] reported their MRI study conducted on 38 patients with chronic coronary diseases that the values of EDV range from $61 \mathrm{ml}$ to $267 \mathrm{ml}$, ESV from $31 \mathrm{ml}$ to $202 \mathrm{ml}$ and EF from 14\% to $59 \%$. Compared to PET where the EDV value is 41 $242 \mathrm{ml}$, ESV 24 - $198 \mathrm{ml}$ and EF 13 - 55 \%. Only ESV showed no significant difference between MRI and PET. Tuncay Hazirolan et al [8] compared their MRI with echocardiography on 20 patients with 15 reported history of myocardial infarction and showed that there is no significant difference between ESV and EF for dilated HF. The EDV and SV values from MRI were higher compared to the measurement from echocardiography. Lissa Sugeng et al [11] compared CT with MRI with 31 subjects (14 female) and found 9 of which were normal and their result showed that the measurement with CT resulted in significantly overestimation of both EDV and ESV. Based on findings from these studies, we found that CMR has no significant difference when compared to PET and echocardiography on LV volumes, but not for CT. EDV, ESV and EF values obtained from our study fall within the range previously reported by other authors $[2,11]$.

\section{CONCLUSION}

This study showed a significant difference on EDV, ESV and EF between normal and HF subjects.

\section{ACKNOWLEDGEMENT}

The first author would like to thank Dr Zhong L. of National Heart Center, Singapore for sharing of his views and interests on the work.

\section{REFERENCES}

[1] L.Z. Barry, M Moser, L.S. Cohen. (1992) Heart book.

[2] H.J.A. S. Riemer, J. B. Jeroen, Richard M. de Jong, Jaep de Boer, Hildo J. Lamb, H. M. Piet, T.M.W. Antoon, Willem Vaalburg, Dirk J. van Veldhuisen, and L. J. Pieter. (2004) "Comparison of Gated PET with MRI for Evaluation of Left Ventricular Function in Patients with Coronary Artery Disease” Journal of Nuclear Medicine;45, 2 176-182. 
[3] S.B. Daniel, R. Hachamovitch, L. J. Shaw, D. F. John, S. W. Hayes, L.E.J. Thomson, S.F. David, G.. Guido, N.D. Wong, X.P. Kang, and A. Rozanski. (2006) "Roles of Nuclear Cardiology, Cardiac Computed Tomography, and Cardiac Magnetic Resonance: Noninvasive Risk Stratification and a Conceptual Framework for the Selection of Noninvasive Imaging Tests in Patients with Known or Suspected Coronary Artery Disease" The Journal of Nuclear Medicine; 47, 1107-1118.

[4] K. Rajappan, L. Livieratos, P.G.. Camici, and D.J. Pennell. (2002) "Measurement of Ventricular Volumes and Function: A Comparison of Gated PET and Cardiovascular Magnetic Resonance” The Journal of Nuclear Medicine; 43, 806-810.

[5] T.R. Miller, J.W. Wallis, B.R. Landy, R.J. Gropler, and C.L. Sabharwal, (1994) "Measurement of Global and Regional Left Ventricular Function by Cardiac PET” The Journal of Nuclear Medicine; 35, 999-1005.

[6] D.S. Berman, R.Hachamovitch, L.J. Shaw, J.D. Friedman, S.W. Hayes, L.E.J. Thomson, D.S. Fieno, G. Germano, P. Slomka, N.D. Wong, X.P. Kang, and A. Rozanski, (2006) "Roles of Nuclear Cardiology, Cardiac Computed Tomography, and Cardiac Magnetic Resonance: Assessment of Patients with Suspected Coronary Artery Disease” The Journal of Nuclear Medicine; 47, 74-82.

[7] Z. Zeidan, R. Erbel, J. Barkhausen, P. Hunold, T. Bartel, and T. Buck, Essen, Germany. (2003) "Analysis of Global Systolic and Diastolic Left Ventricular Performance Using Volume-time Curves by Real time Three Dimensional Echocardiography”J Am Soc Echiocardiogr;16:29-37.

[8] T. Hazirolan, B. Tasbas, M.G. Dagoglu, M. Canyigit, G. Abali, K. Aytemir, A. Oto, F. Balkanci. (2007) "Comparison of short and long axis methods in cardiac MR imaging and echocardiography for left ventricular function” Diagn Interv Radiol; 13, 33-38.

[9] W.M. Schaefer, C.S.A. Lipke, D. Standke, H.P. Kühl, B. Nowak, H.J. Kaiser, K.C. Koch, and U. Buell. (2005) “Quantification of Left Ventricular Volumes and Ejection Fraction from Gated 99mTc-MIBI SPECT: MRI Validation and Comparison of the Emory Cardiac Tool Box with QGS and 4D-MSPECT” The Journal of Nuclear Medicine; 46, 1256-1263.

[10] B.J. Krenning, M.M. Voormolen and J.R.T.C. Roeland. (2003) "Assessment of Left ventricular function by Three- dimensional Echocardiography Department of Cardiology. Cardiovasc Ultrasound. 1:12.

[11] L. Sugeng, V.M. Avi, L. Weinert, N. Johannes, E. Christian, R. Steringer-Mascherbauer, S. Frank, G. Christian, S. George; R.M. Lang, H.J. Nesser, (2006) "Quantitative Assessment of Left Ventricular Size and Function” Circulation;114:654-661.

[12] F. Roman, J. Kai; O. Murat; M. David; Grude, Matthias; Seifarth, Harald; Heindel, Walter; Wichter, Thomas. (2007) “Assessment of regional left ventricular function with multidetector-row computed tomography versus magnetic resonance imaging” European Radiology;17, 1009- 1017. 